\title{
Distribución y variación morfológica de Amphiroa beauvoisii (Corallinales, Rhodophyta) en México
}

\author{
Distribution and morphological variation of Amphiroa beauvoisii (Corallinales, Rhodophyta) \\ in Mexico
}

\author{
Luz Elena Mateo-Cid ${ }^{1 *}$, Raúl Aguilar-Rosas², A. Catalina Mendoza-González¹, Luis E. Aguilar-Rosas ${ }^{3}$ \\ ${ }^{1}$ Departamento de Botánica, Escuela Nacional de Ciencias Biológicas, Instituto Politécnico Nacional. Carpio y Plan de ayala s/n, Col. Santo Tomás \\ 11340 México, D.F., México \\ ${ }^{2}$ Facultad de Ciencias Marinas, Universidad Autónoma de Baja California, Km 107 Carr. Tijuana-Ensenada, Ensenada, Baja California, México \\ ${ }^{3}$ Instituto de Investigaciones Oceanológicas, Universidad Autónoma de Baja California, Km 107 Carr. Tijuana-Ensenada, Ensenada, Baja California, \\ México \\ *Correspondencia:lmateoc@ipn.mx
}

\begin{abstract}
Resumen. Se realizaron muestreos de Amphiroa beauvoisii (Corallinales, Rhodophyta) en diversas localidades de las costas del Pacífico y Caribe mexicano de 1994 a 2007 y se efectuó una extensa revisión bibliográfica de los trabajos en los que se cita Amphiroa beauvoisii. Asimismo, se revisaron los ejemplares depositados en herbarios nacionales y extranjeros, con el propósito de determinar la distribución latitudinal, y la variación morfológica y reproductiva de esta especie. El análisis de la información nos indica que se encuentra ampliamente distribuida en la costa del Pacífico desde islas Coronados, Baja California hasta La Ventosa, Oaxaca, siendo muy frecuente en el golfo de California y en los estados de Jalisco, Guerrero y Oaxaca, mientras que en la costa atlántica su distribución se encuentra restringida a los arrecifes coralinos de Yucatán y Quintana Roo. Se describen en forma detallada los caracteres morfo-anatómicos y reproductivos. En general se observó que los talos tetrasporangiales y bisporangiales son frecuentes, de mayor talla y más ramificados que las plantas gametangiales. El material examinado mostró que en la costa del Pacifico se encuentran los talos tetrasporangiales, bisporangiales y gametangiales, mientras que en la costa del Caribe mexicano sólo se presentan poblaciones tetrasporangiales. Se registra por primera vez la fase gametofitica masculina y femenina de A. beauvoisii en poblaciones mexicanas.
\end{abstract}

Palabras clave: Amphiroa, coralina articulada, variación morfológica, distribución, Pacífico, Caribe mexicano.

\begin{abstract}
Several samples of Amphiroa beauvoisii (Corallinales, Rhodophyta) were collected in different localities along the Pacific and Caribbean coasts of Mexican from 1994 to 2007. An extensive review of Amphiroa beauvoisii recorded from Mexico was carried out. Specimens of different herbaria were examined to determine its geographic distribution, as well as morphological and reproductive data for this taxon. Analysis of this information showed that this species is distributed along the Pacific coast from Islas Coronados, Baja California to La Ventosa, Oaxaca. A. beauvoisii is frequent in the Gulf of California and Jalisco, Guerrero and Oaxaca, while on the Atlantic coast it is rare and we only found it on coralline reefs of Yucatán and Quintana Roo. Morphological variation, reproductive stage, and distinctive characteristics of this taxon are included. A. beauvoisii is an extremely variable species; the tetrasporangial and bisporangial thalli are more common, more branched and taller that the gametangial plants. We found gametangial and tetrasporangial plants on the Pacific coast and only tetrasporangial plants in the Caribbean. Male and female plants of A. beauvoisii are described for the first time from Mexican populations.
\end{abstract}

Key words: Amphiroa, articulated Coralline, morphological variation, distribution, Pacific, Caribbean, Mexico.

\section{Introducción}

El género Amphiroa J.V. Lamouroux (1812) es una coralina articulada que se distribuye ampliamente en las regiones tropicales y subtropicales del mundo (Norris

Recibido: 06 diciembre 2006; aceptado: 20 noviembre 2007 y Johansen, 1981) y tiene una gran importancia en la formación de arrecifes coralinos (Dolan, 2001). Este género se caracteriza por la anatomía de las frondas ramificadas que parten de una porción basal costrosa, están formadas por una sucesión de genículas e intergenículas, existe una gran variedad morfológica en los talos y estos miden desde pocos milímetros hasta 30 centímetros de 
alto. La intergenícula está impregnada con carbonato de calcio, puede ser de cilíndrica a comprimida y consta de una médula en la que alternan filamentos de células largas y cortas que derivan de un tejido meristemático y una corteza con número variable de capas. La genícula, que es la porción no calcificada del talo, está constituida por numerosas capas de células que forman filamentos (Dolan, 2001). En el mundo, Amphiroa está representado por 45 especies (Guiry y Guiry, 2006); en México es el género más frecuente en las costas rocosas del Pacífico y se encuentra ocasionalmente en los arrecifes del Caribe mexicano (Mateo-Cid y Mendoza-González, 1991, 2001; González-González et al., 1996; Ortega et al. 2001). En las costas de México se tienen registradas 9 especies, de las cuales $A$. beauvoisii J.V. Lamouroux es la que presenta la mayor distribución (Dawson, 1953; Norris y Johansen, 1981; González-González et al., 1996; Ortega et al., 2001).

No obstante, el número real de especies de Amphiroa aún es controversial y los problemas taxonómicos de este género son significativos debido a su plasticidad morfológica (Riosmena-Rodríguez y Siqueiros-Beltrones, 1996). Al realizar la revisión de ejemplares depositados en herbarios y los recolectados recientemente en México, se observó que existían variaciones morfológicas entre los talos de A. beauvoisii, por lo que se consideró importante estudiar especímenes que representaran los caracteres morfológicos y reproductivos que delimitan esta especie, así como los que son altamente variables. Con este propósito se realizó la revisión de ejemplares de herbario de $A$. beauvoisii, así como de la bibliografía y de los ejemplares obtenidos en el campo, y con ello contribuir al conocimiento sobre la distribución y variación morfológica de $A$. beauvoisii en las costas de México.

\section{Materiales y método}

Se realizaron 15 campañas de recolección durante 1996, 1997, 1998 y 2007 en las que se obtuvieron 50 muestras de $A$. beauvoisii en 18 localidades de la costa del Pacífico y Caribe de México. Los muestreos se llevaron a cabo en las zonas rocosas de los niveles del intermareal durante las bajamares y por buceo libre en el submareal hasta los $4 \mathrm{~m}$ de profundidad. Las 5 recolectas de material ficológico realizadas para este estudio fueron ocasionales y se llevaron a cabo de 1994 a 2007. Los ejemplares recolectados se preservaron en formaldehído al $4 \%$ en agua de mar, se etiquetaron, se colocaron en bolsas de plástico y se trasladaron al laboratorio de Ficología de la Escuela Nacional de Ciencias Biológicas para su procesamiento. Se efectuó una amplia revisión de la literatura relacionada con la familia Corallinaceae con el fin de obtener registros geográficos e información general de $A$. beauvoisii. Con el objeto de corroborar, corregir las determinaciones taxonómicas y obtener registros geográficos de los ejemplares depositados en los herbarios, se revisaron los ejemplares de $A$. beauvoisii depositados en diversos herbarios ficológicos (Holmgren et al., 1990): Herbario de la Escuela Nacional de Ciencias Biológicas (ENCB), Herbario de la Facultad de Ciencias Marinas (CMMEX), Herbario del Departamento de Biología Marina de la Universidad Autónoma de Baja California Sur (FBCS), Herbario de la Universidad Autónoma MetropolitanaIztapalapa (UAMIZ) y Herbario del Museo de Historia Natural de los Ángeles (LAM). Así como el espécimen tipo de A. mexicana Taylor (LAM 3826).

Para la descripción de la especie y el análisis de la variabilidad de los caracteres morfológicos y reproductivos se seleccionaron 125 especímenes de los 256 ubicados en los herbarios y los recolectados durante las campañas de muestreo. Los caracteres considerados para los esporofitos fueron: talla, forma y longitud/ancho o diámetro de la intergenícula media y apical, longitud/diámetro de las células medulares, número de capas de la corteza y de la genícula, diámetro/alto de las cámaras tetrasporangiales y de los tetrasporangios. Se realizaron cortes histológicos transversales y longitudinales de las estructuras vegetativas y reproductivas, de cada carácter se tomaron 30 medidas. Los especímenes fueron descalcificados con $0.6 \mathrm{M} \mathrm{HNO}^{3}$ y deshidratados con alcohol etílico. Se montaron en parafina pequeños segmentos y se cortaron en secciones de 9-10 $\mu \mathrm{m}$ de grosor con ayuda de un micrótomo de mano (American Optical, EUA), se fijaron con pegamento Ruyter`s (Martoja y Martoja-Pierson, 1970) y se tiñeron con anilina azul y hematoxilina-eosina para el análisis posterior de las características morfo-anatómicas de los talos. Las fotografías se tomaron con una cámara digital (Sony DSC-S85, Japón) en un microscopio Zeiss (Axioscop 40, Goettingen, Alemania). Las figuras fueron editadas usando Adobe ${ }^{\circledR}$ Photoshop ${ }^{\circledR} 7.0$ (Adobe Systems, EUA). Las preparaciones permanentes y el material recolectado quedaron depositados en el Herbario ENCB. Se siguió la terminología descriptiva establecida por Norris y Johansen (1981) y Moura y Beauclair (2005). Para definir la distribución de $A$. beauvoisii en la costa de México, se consideraron las regiones geográficas establecidas por Pedroche y Sentíes (2003).

\section{Descripción}

Amphiroa beauvoisii J.V. Lamouroux, 1816: 299-300 (Localidad tipo: Portugal) 
Sinónimos taxonómicos considerados previamente por Norris y Johansen, 1981 y Riosmena y SequeirosBeltrones, 1996:

A. linearis Kützing, 1858: 22, pl. 46

A. zonata Yendo, 1902: 10, pl. 1: figs. 11-14.

A. pusilla sensu Dawson, 1944: 276 (en parte) (no $A$. pusilla Yendo, 1902:13).

A. mexicana W. Taylor, 1945: 189 (Localidad tipo: Petatlán, Guerrero, México).

A. peninsularis W. Taylor, 1945: 188 (Localidad tipo: Isla Cedros, B.C., México).

A. crosslandii sensu Dawson, 1953: 149; (no A. crosslandii Lemoine, 1929:50).

A. drouetii E.Y. Dawson, 1953: 140, pl. 27 (Localidad tipo: Isla Turner, Sonora, México).

A. franciscana var. robusta E.Y. Dawson, 1953: 150 (Localidad tipo: Acapulco, Guerrero, México).

Morfología y anatomía vegetativa. Talos de hábito erecto de color rosa-verdoso a guinda, con una fuerte impregnación de carbonato de calcio, de 2.5 a $6.0 \mathrm{~cm}$ alto, fijos al sustrato por un disco costroso pequeño y poco evidente, ramificación predominantemente dicotómica, algunas veces tiende a ser subdicotómica, formando densos agregados de $0.1-4.0 \mathrm{~cm}$. de ancho; en las porciones basales las intergenículas cilíndricas a comprimidas de 0.8 a $1.0 \mathrm{~mm}$ de diámetro, algunas veces de apariencia zonada, en las porciones medias las intergenículas subcilíndricas a comprimidas. En sección longitudinal intergenícula de 600-800 $\mu \mathrm{m}$ de diámetro, la médula está constituida por 3-6 capas de células largas alternando con una capa de células cortas, las largas de 36-77 $\mu \mathrm{m}$ de largo por 9-12 $\mu \mathrm{m}$ de diámetro y las cortas de $9-17 \mu \mathrm{m}$ de alto por 8-10 $\mu \mathrm{m}$ de diámetro (Fig. 16), la corteza formada por 4-7 capas de células de 4-7 $\mu \mathrm{m}$ de diámetro por 10-12 $\mu \mathrm{m}$ de largo, epitalo de 1 capa de células oblongas de 1.5-2.5 $\mu \mathrm{m}$ de alto por 5-8 $\mu \mathrm{m}$ de ancho (Fig. 17). Genícula multizonada con 3-8 capas de células, algunas veces corticada e impregnada con $\mathrm{CaCO}_{3}$; de 450-600 $\mu \mathrm{m}$ ancho, visible a simple vista (Fig. 15).

Reproducción. Gametofito masculino. Talos de 1.2-3.5 $\mathrm{cm}$. de alto, ramificación dicotómica a subdicotómica, intergenículas cilíndricas en la base, cilíndricas a comprimidas en la porción media de 2-3 $\mathrm{mm}$ de longitud por $675 \mu \mathrm{m}-1.0 \mathrm{~mm}$ de diámetro, tendiendo a comprimirse hacia los ápices, en donde miden de 1-4 $\mathrm{mm}$ de longitud por $0.76-2.0 \mathrm{~mm}$ de ancho (Fig. 7). En sección longitudinal, médula constituida por 3 a 5 capas de células largas de 5.7-8.4 $\mu \mathrm{m}$ de diámetro y 74-111 $\mu \mathrm{m}$ de longitud que alternan con una capa de células cortas que miden 5.7-8.1 $\mu \mathrm{m}$ de diámetro y 30-40 $\mu \mathrm{m}$ de longitud, corteza constituida por 4-13 capas de células, éstas de
4-6.0 $\mu \mathrm{m}$ de diámetro y 9-14 $\mu \mathrm{m}$ de alto, las epiteliales de 13-15 $\mu \mathrm{m}$ de ancho y 4-6.5 $\mu \mathrm{m}$ de alto (Figs. 3, 5). Genícula constituida por 4 capas de células no corticadas. Conceptáculos espermatangiales corticales, numerosos por intergenícula, cámaras comprimidas a oblongas de 230-310 $\mu \mathrm{m}$ de diámetro y 148-190 $\mu \mathrm{m}$ de alto (Fig. 14), espermatangios dispuestos sobre el piso de las cámaras, espermacios de $1 \mu \mathrm{m}$ de diámetro.

Material examinado. Punta Conejo, Salina Cruz, Oaxaca, 7-VIII-1992 (ENCB 8969); Bahía Tangolunda, Oaxaca; 8VIII-1993 (ENCB 8979).

Gametofito femenino. Talos de $2-4.0 \mathrm{~cm}$ de alto, ramificados dicotomicamente (Fig. 8), intergenículas cilíndricas en la base, comprimidas en la parte media en la que alternan 3 a 4 capas de células largas de $550 \mu \mathrm{m}-3 \mathrm{~mm}$ de longitud por 1.0-1.1 mm de ancho, con 1 capa de células cortas de 7-8.0 $\mu \mathrm{m}$ de diámetro y 15-20 $\mu \mathrm{m}$ de longitud, cilíndricas en la parte apical de $3-3.5 \mathrm{~mm}$ de longitud por $0.5 \mathrm{~mm}$ de diámetro, en sección longitudinal las células medulares de la intergenícula media miden 7.8-8.4 $\mu \mathrm{m}$ de diámetro por 92.5-108 $\mu \mathrm{m}$ de longitud por 7.4-8.5 $\mu \mathrm{m}$ de diámetro, la genícula constituida por 3 a 5 capas de células, la intergenícula constituida por una médula en la que alternan 3 a 4 capas de células largas de 7-8.0 $\mu \mathrm{m}$ de diámetro y $60-75 \mu \mathrm{m}$ de longitud, la corteza constituida por 5-10 capas de células, éstas de 7-9.0 $\mu \mathrm{m}$ de diámetro y 4-6.0 $\mu \mathrm{m}$ de alto, una capa de células epiteliales, que miden12$14 \mu \mathrm{m}$ de diámetro y 4-6.0 $\mu \mathrm{m}$ de alto. Conceptáculos embebidos en la corteza (Fig. 8), carpogonios numerosos en el piso de la cámara sin columnella (Fig. 12), cámaras orbiculares de 240-270 $\mu \mathrm{m}$ de diámetro y 92.5-160 $\mu \mathrm{m}$ de alto, carposporangios de 18-21 $\mu \mathrm{m}$ de diámetro y 28-40.0 $\mu \mathrm{m}$ de longitud (Fig. 13).

Material examinado. Bahía de Cuastecomate, Jalisco, 15VII-1991 (ENCB 8956); Puerto Escondido, Oaxaca; 30IX-1998 (ENCB 8983).

Tetrasporofito. Talos de 1.5-10 cm de alto, con variación morfológica notable, algunos talos poco ramificados pero la mayoría muy ramificados, la ramificación varía de dicotómica a irregular. Intergenículas cilíndricas en la base, cilíndricas a comprimidas en las partes medias, de 1.0-7.0 $\mathrm{mm}$ de longitud por 0.4-2 $\mathrm{mm}$ de ancho/diámetro (Figs. 4, 5, 6), intergenícula de los ápices de 1-6 mm de longitud por $0.3-1.5 \mathrm{~mm}$ de diámetro, constituida por 2-6 capas de células medulares largas de 48-118 $\mu \mathrm{m}$ de longitud por 7-12.5 $\mu \mathrm{m}$ de diámetro, alternando con una capa de células cortas de 9-20 $\mu \mathrm{m}$ de alto por 8-10 $\mu \mathrm{m}$ de diámetro, la corteza formada por 2-9 capas de células de 4-7 $\mu \mathrm{m}$ de diámetro por 10-12 $\mu \mathrm{m}$ de largo, epitalo de 1 capa de células oblongas de $1.5-2.5 \mu \mathrm{m}$ de alto por 5-8 $\mu \mathrm{m}$ ancho. Genícula multizonada con 2-10 capas de células, corticada o no; de 450-600 $\mu \mathrm{m}$ de ancho, visible a simple 


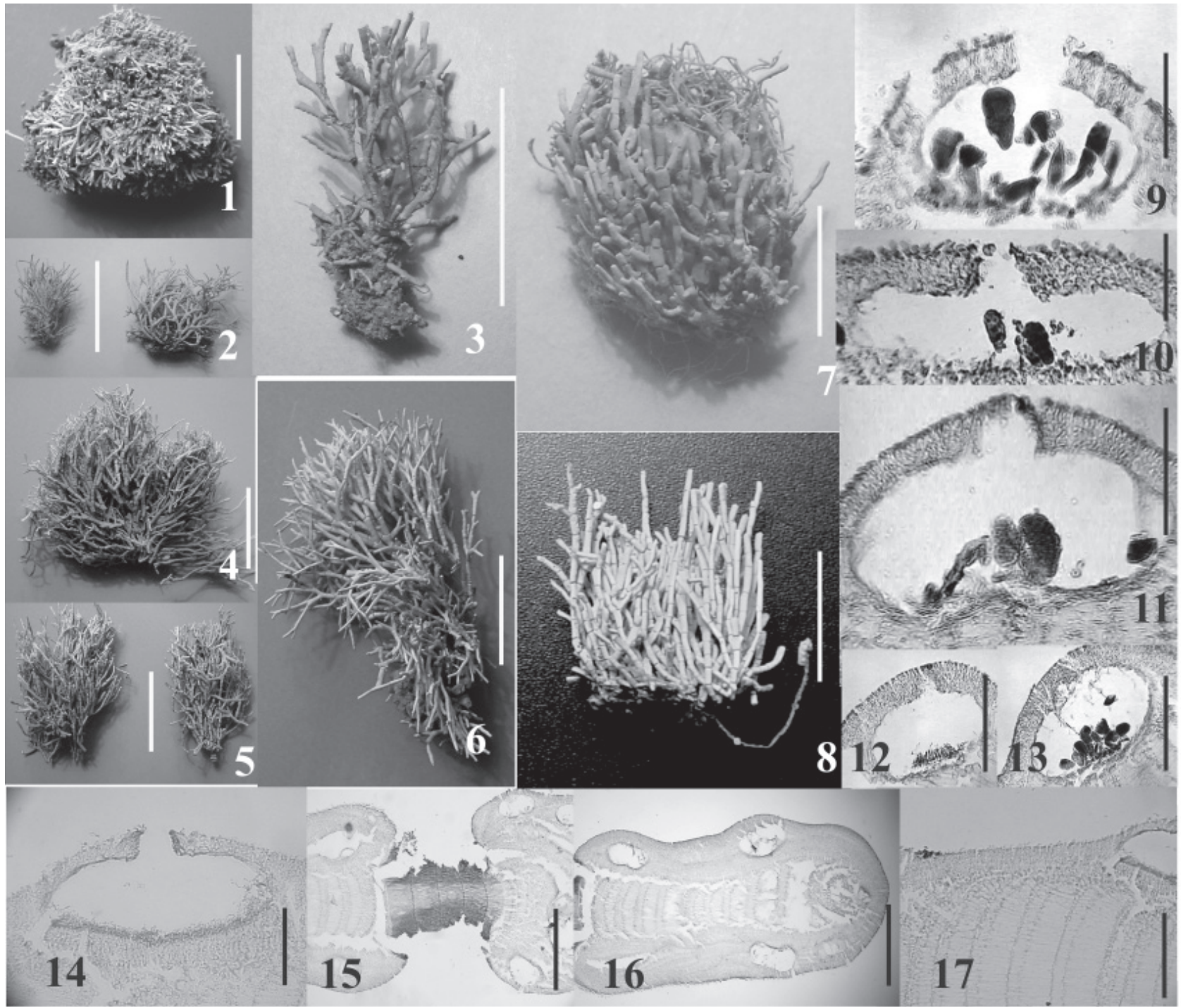

Figuras 1-17. Amphiroa beauvoisii. 1. Hábito de la planta bisporangial. (ENCB 8914). Playa Santa Teresa, Baja California, 4 de febrero de 1995. 2. Hábito de la planta bisporangial (ENCB 7998). Puertecitos, Baja California, 22 de octubre 1995. 3. Hábito de la planta bisporangial (ENCB 8954). Careyes, Jalisco, 13 de julio de 1992. 4. Hábito de la planta tetrasporangial (ENCB 7996). Las Palmas, Bahía Concepción, Baja California Sur, 23 de mayo de 1999. 5. Hábito de la planta tetrasporangial (ENCB 7996). 6. Hábito de la planta tetrasporangial (ENCB 12217). Isla Ixtapa, Playa Coral, Guerrero, Oaxaca, 7 de noviembre de 1996. 7. Hábito de la planta espermatangial (ENCB 8969). Punta Conejo, Salina Cruz, Oaxaca, 7 de agosto de 1992. 8. Hábito de la planta carposporangial (ENCB 8956). Bahía de Cuastecomate, Jalisco, 15 de julio de 1991. 9. Detalle del conceptáculo bisporangial (ENCB 8914). 10. Detalle del conceptáculo tetrasporangial (ENCB 7996). 11. Detalle del conceptáculo tetrasporangial (ENCB 7996). 12. Detalle del conceptáculo con carpogonios (ENCB 8956). 13. Detalle del conceptáculo carposporangial (ENCB 8956). 14. Detalle del conceptáculo espermatangial (ENCB 8969). 15. Detalle de la genícula (ENCB 8914). 16. Detalle de una intergenícula apical (ENCB 8914). 17. Sección vertical de las células corticales y medulares de la intergenícula (ENCB 8969). Escalas, 1-6: 2 cm, 7 y 8: 1 cm, 9-14: 100 $\mu$ m, 15 y $16: 400 \mu \mathrm{m}, 17: 200 \mu \mathrm{m}$

vista. Conceptáculos uniporados, corticales, embebidos en la corteza de toda la intergenícula (Fig. 10) o proyectados. Cámaras tetrasporangiales de 148-300 $\mu \mathrm{m}$ de diámetro y 74-150 $\mu \mathrm{m}$ de alto (Figs. 10, 11), tetrasporangios zonados de 35-92 $\mu \mathrm{m}$ de alto y 18.5-40 $\mu \mathrm{m}$ de diámetro.
Bisporofito. Talos de 1.2-6.0 cm. de alto, ramificación subdicotómica, intergenículas cilíndricas y comprimidas en la base y en las porciones medias y algunas veces cilíndricas en los ápices (Figs. 1, 2, 3), intergenículas en las porciones medias de 1-7 $\mathrm{mm}$ de longitud por 0.5- 
$2.0 \mathrm{~mm}$ de ancho y en las partes apicales de 1-7 mm de longitud por 0.3-1.0 mm de ancho. En sección longitudinal intergenículas de 750-840 $\mu \mathrm{m}$ de diámetro, médula de células alargadas de 7.4-11 $\mu \mathrm{m}$ de diámetro y 64-125 $\mu \mathrm{m}$ de longitud, estas capas largas alternan con capas de células cortas de 7.0-7.5 $\mu \mathrm{m}$ de diámetro y 30-35 $\mu \mathrm{m}$ de longitud, corteza constituida por 2-8 capas de células, éstas miden 11-12.5 $\mu \mathrm{m}$ de diámetro y 12-17.5 $\mu \mathrm{m}$ de largo, células epiteliales de 7.5-8.0 $\mu \mathrm{m}$ de ancho y 3-4 $\mu \mathrm{m}$ de alto. Conceptáculos bisporangiales embebidos en la corteza y en ocasiones proyectados, cámaras alargadas u oblongas de 240-480 $\mu \mathrm{m}$ diámetro y (70)105-150 $\mu \mathrm{m}$ alto, bisporangios de 22-46 $\mu \mathrm{m}$ de diámetro y 40-80 $\mu \mathrm{m}$ de longitud (Fig. 9).

Material seleccionado. BAJA CALIFORNIA (PACÍFICO): Islas Coronado, 20-VI-1946, E. Y. Dawson (LAM 19502), 14-VIII-1995, R. Riosmena, (FBCS 2385; Dawson, 1953: 146-148, como A. zonata; Norris y Johansen, 1981: 6-12). Bahía de Todos Santos y alrededores (Devinny, 1978: 360, Mendoza-González y Mateo-Cid, 1985: 26, ambos como A. zonata); Ensenada, Marina Coral Sur, 2-VIII-1996, A. C. Mendoza (ENCB 12211); Punta Santa Rosaliita (Dawson, 1953: 146-148, como A. zonata; Norris y Johansen, 1981: 6-12); Punta Santa Rosalía (Dawson, 1953: 146-148 como A. zonata). Bahía Santa Rosalía (Littler y Littler; 1981: 150, como A. zonata; Campo Diamante, 31-VII-1996, A. C. Mendoza-González y L. E. Mateo-Cid (ENCB 12227); Campo San José, 31VIII-1996, A. C. Mendoza-González y L. E. Mateo-Cid (ENCB 12229); Desembarcadero de Miller (Dawson, 1949: 228, como A. peninsularis; Dawson, 1953: 146-148, como A. zonata; Norris y Johansen, 1981: 6-12); Isla San Benito (Dawson, 1951: 53 como A. peninsularis); Isla Cedros 30-X-1951, C. L. Hubbs (LAM 57957), 19-IV1950, E.Y. Dawson (LAM 57250), 5-III-1949, E. Y. Dawson (LAM 501351) (Taylor, 1945: 188 como $A$. peninsularia; Dawson, 1953: 146-148 como A. zonata, Norris y Johansen, 1981: 6-12); Isla Guadalupe, 18-XII1949, E. Y. Dawson (LAM 55148) (Dawson, 1953: 146148 como A. zonata; Norris y Johansen, 1981: 6-12). BAJA CALIFORNIA SUR (PACÍFICO): Bahía Sebastián Vizcaíno (Norris y Johansen, 1981: 6-12); Punta Malarrimo (Norris y Johansen 1981: 6-12); Punta Malarrimo (11 millas al oeste) 17-IV-1951, E. Y. Dawson (LAM 57395) (Dawson, 1953: 146-148, como A. zonata); Punta Eugenia (Riosmena-Rodríguez y Siqueiros-Beltrones, 1996: 137138), 31-X-1951, E. Y. Dawson (LAM 501342) (Dawson, 1953: 146-148; Dawson et al., 1960: 8, ambos como $A$. zonata; Norris y Johansen; 1981: 6-12); Bahía Tortugas, 7-III-1949, E.Y. Dawson (LAM 55107), 24-VI-1983, A.C. Mendoza-González (ENCB 8913), 14-VI-1986, A. C. Mendoza-González (ENCB 8924), 27-III-1986, V.
Anderson, (LAM 502595), 18-XI-1986, S.A. Guzmán del Próo (ENCB 7986), 19-V-1988, S.A. Guzmán del Próo (ENCB 8927); Bahía San Bartolomé (Norris y Johansen 1981: 6-12); Punta Thurloe (Norris y Johansen, 1981: 612); Punta Asunción (Dawson et al. 1960: 10 como $A$. zonata); Puerto Asunción 7-I-1989, R. Riosmena (FBCS 2004); 28-IX-1989, R. Riosmena (FBCS 2005); Isla Asunción, 12-I-1989, R. Riosmena (FBCS 2006), 28-IX1989, L.E. Mateo-Cid y M. Aguirre (ENCB 7988) (Dawson et al., 1960: 11, como A. zonata; Norris y Johansen, 1981: 6-12; Mateo-Cid y Mendoza-González, 1994a: 47); Bahía Asunción, 20-IV-1950, E.Y. Dawson (UC 974629), 28IV-1950, E. Y. Dawson (LAM 54314), 26-VII-1989, L. E. Mateo-Cid y M. Aguirre (ENCB 8907) (Dawson, 1951: 53, como A. peninsularis; Dawson, 1953: 146-148, como A. zonata Norris y Johansen, 1981: 6-12); Bahía San Hipólito (Dawson et al. 1960: 11 como A. zonata; Norris y Johansen, 1981: 6-12) Punta Abreojos, 30-IV-1950, E. Y. Dawson (LAM 54512) (Dawson et al. 1960: 12, Dawson, 1953: 146-148, ambos como A. zonata; Norris y Johansen, 1981: 6-12; Laguna de San Ignacio, 21-V-1992, R. A. Núñez y M. Casas (ENCB 8903), 5-XI-1992, Ma. Casas y R. A. Núñez (ENCB 8919) (Núñez-López et al. 1998: 36); Isla Magdalena, 8-III-1949, E. Y. Dawson (LAM 501354), 7-XI-1982, M. Aguirre (ENCB 12471), 9-XII-1987, E. Rodríguez (ENCB 8921) 9-XI-1984, I. Sánchez (ENCB 8904) (Dawson, 1951: 53 como A. peninsularis; Dawson, 1953: 146-148, como A. zonata; Riosmena-Rodríguez y Siqueiros Beltrones, 1996: 137-138), Bahía Magdalena, 22-IX-1985, I. Sánchez y M. Aguirre (ENCB 7987), 25XI-1985, I. Sánchez y M. Aguirre (ENCB 7984) (Dawson, 1953: 146-148, como A. zonata; Norris y Johansen, 1981: 6-12; Sánchez-Rodríguez et al., 1989: 42 como A. drouetii; Sánchez-Rodríguez et al., 1989: 36, 38, 42 como $A$. zonata); Punta Entrada, 2-V-1950, E. Y. Dawson (LAM 54395); Isla Santa Margarita, 9-III-1949, E.Y. Dawson (LAM 501353) (Riosmena-Rodríguez y SiqueirosBeltrones, 1996: 137-138; Punta Conejo, 5-XII-1992, L. E. Mateo-Cid y C. Mendoza-González (ENCB 7993); Punta Márquez (Littler y Arnold, 1982: 309, como $A$. zonata); Rocas Alijos, 9-X-1956, E. Y. Dawson (LAM 48209); Los Cerritos (Todos Santos), 12-III-1987, R. Riosmena (FBCS 2003), 12-XI-1988, L. E. Mateo-Cid y M. Aguirre (ENCB 8916), 31-V-1989, L. E. Mateo-Cid y M. Aguirre (ENCB 7990), 4-VIII-1989, L. E. Mateo-Cid y G. Garduño (ENCB 8925), 12-XI-1989, L. E. Mateo-Cid y C. Mendoza-González (ENCB 7989), 30-V-1994, R. Riosmena (FBCS 877) (Mateo-Cid y Mendoza-González, 1994b: 39); Las Cabrillas (Todos Santos) (RiosmenaRodríguez y Siqueiros-Beltrones, 1996: 137-138). BAJA CALIFORNIA (GOLFO): Campo Turístico Vallarta, 31VII-1996, A. C. Mendoza-González y L. E. Mateo-Cid 
(ENCB 12228), Playa San Antonio, 31-VII-1996, A. C. Mendoza-González, L. E. Mateo-Cid (ENCB 12213); El Coloradito, 5-II-1996, L. E. Mateo-Cid, A. C. MendozaGonzález, L. E. Aguilar y R. Aguilar-Rosas (ENCB 12211), 22-X-1995, C. Mendoza-González, L. E. AguilarRosas, L. E. Mateo-Cid y R. Aguilar-Rosas (ENCB 8922), Playa Santa Teresa, 4-II-1995, L. E. Aguilar-Rosas, R. Aguilar-Rosas, A. C. Mendoza-González y L. E. MateoCid (ENCB 8914), 30-VII-1996, A. C. Mendoza-González, L. E. Mateo-Cid, R. Aguilar-Rosas y L. E. Aguilar-Rosas (ENCB 12212); (Aguilar-Rosas et al. 2000: 130); Puertecitos, 3-V-1996, R. Aguilar-Rosas, L. E. AguilarRosas, A. C. Mendoza-González y L. E. Mateo-Cid (ENCB 8911), 30-VII-1996, L. E. Mateo-Cid, A. C. MendozaGonzález, R. Aguilar-Rosas y L. E. Aguilar-Rosas (ENCB 12214); Punta Bufeo (Littler y Littler, 1981: 51 como $A$. zonata) Isla Ángel de la Guarda, 26-I-1940, E. Y. Dawson (LAM 1551), Puerto Refugio (Dawson, 1944: 276 como A. zonata; Dawson, 1944: 276 (pro parte) como A. pusilla; Dawson, 1953: 146-148, como A. zonata; Norris y Johansen, 1981: 6-12); Islas Gemelos (Norris y Johansen, 1981: 6-12); Bahía de los Ángeles (Norris y Johansen, 1981: 6-12); Isla San Pedro Mártir (Riosmena-Rodríguez y Siqueiros-Beltrones, 1996: 137-138). BAJA CALIFORNIA SUR (GOLFO). Isla Tortuga (Dawson, 1959:10, 20, 22 como A. zonata); Bahía Concepción, El Coyote, 24-X-1990, R. Riosmena (FBCS 2099), El Manglito, 13-III-1990, L. E. Mateo-Cid e I. Sánchez (ENCB 8905); El Requesón, R. Riosmena (FBCS 2101), La Calavera, 17-I-1990, E. Rodríguez (ENCB 8909); 23V-1990, I. Sánchez y M. Aguirre (ENCB 7983); La Tasajera, 16-I-1990, I. Sánchez (ENCB 8906). Las Palmas, 17-I-1990, L.E. Mateo-Cid y E. Rodríguez (ENCB 7997); 23-V-1990, L.E. Mateo-Cid y M. Aguirre (ENCB 7996); Santispack, 18-I-1990, L. E. Mateo-Cid e I. Sánchez (ENCB 8923), Punta Santa Rosaliita, L. E. Mateo-Cid e I. Sánchez (ENCB 8912), Punta Morales, 23-V-1990, L. E. Mateo-Cid y M. Aguirre (ENCB 7999), (Dawson, 1953: 146-148 como A. zonata; Norris y Johansen, 1981: 6-12; Mateo-Cid et al. 1993: 46), Isla San Ildefonso (Norris y Johansen, 1981: 6-12); Isla Cholla, 23-IV-1958, E. Y. Dawson (LAM 52748); (Dawson, 1959: 8, 20,22 como $A$. zonata, Norris y Johansen, 1981: 6-12); Puerto Balandro (Dawson, 1959: 8, 20, 22 como A zonata); Isla del Carmen, 21-III-1949, E. Y. Dawson (LAM 501348) (Dawson, 1953: 146.148 como A. zonata); Puerto Escondido (Dawson, 1959: 7, 20, 22 como A. zonata); Isla Montserrat, 21-IV-1958, E. Y. Dawson (LAM 52701) (Dawson 1959: 7, 21 como $A$. drouetii; Dawson, 1959: 6-20, 22, como $A$. zonata, Norris y Johansen, 1981: 6-12); Isla San Diego, 19-IV-1958, E.Y. Dawson (LAM 52901) (Dawson, 1959: 5, 21 como A. drouetii); El Solitario (Dawson, 1959: 6, 20,
22 como A. zonata); Bahía Agua Verde, 12-II-1940, E. Y. Dawson (LAM 57771), 2-II-1985, H. Chaney (LAM 502518) (Dawson, 1953: 150 como A. franciscana var. robusta; Dawson, 1953: 146-148, como A. zonata; Dawson, 1959: 6, 21 como A. drouetii; Norris y Johansen, 1981: 612), Bahía de La Paz: El Tecolote, 13-V-1984, R. Riosmena (FBCS 1997); San Juan de la Costa, 22-V-1988, R. Riosmena (FBCS 877); Malecón de la ciudad, 10-XI-1946, E. Y. Dawson (LAM 55178); Balandra, 30-I-1988, R. Riosmena (FBCS 1871), 6-V-1988, R. Riosmena (FBCS 1877), 24-IV-1982, R. Riosmena (FBCS 1987); 27-X1989, R. Riosmena (FBCS 1911); 8-5-1990, R. Riosmena (FBCS 2051), 26-II-1990, R. Riosmena (FBCS 2017); 21X-1989, A. C. Mendoza-González (ENCB 7992); Calerita, 16-X-1979, M. M. Casas y S. Hernández, (ENCB 8915), 21-X-1989, A.C. Mendoza-González y L. E. Mateo-Cid (ENCB 8901), 31-V-1988, R. Riosmena (FBCS 1929), $17-$ XI-1989, R. Riosmena (FBCS 1984), 8-V-1990, R. Riosmena (FBCS 2076); 24-VIII-1990, R. Riosmena (FBCS 2081); 26-IV-1991, R. Riosmena (FBCS 2151), 7V-1994, G. Anaya (FBCS 724); 22-II-1991, R. Riosmena (FBCS 1956) (Dawson, 1953: 148-149, como $A$. crosslandii; Dawson, 1959: 8, 20, 22, como A. zonata; Norris y Johansen, 1981: 6-12, Huerta-Múzquiz y Mendoza-González, 1985: 50 como A. zonata; SánchezCastrejón et al. 1995: 323); Isla Partida, 9-V-1990, R. Riosmena (FBCS 2065), 20-IX-1990, R. Riosmena (FBCS 2091), Isla Espíritu Santo (Punta Prieta), 20-II-1991, R. Riosmena (FBCS 2138), (San Gabriel), 14-III-1949, E. Y. Dawson (LAM 501349), 9-V-1990, R. Riosmena (FBCS 2061), 20-IX-1990, R. Riosmena (FBCS 2095), Isla Cerralvo, 10-V-1994, Anaya y H. Morsaria (FBCS 749); Punta Arena (Mateo-Cid et al. 2000: 10); El Cardonal, 21XI-1996, A. C. Mendoza-González y L.E. Mateo-Cid (ENCB 12230); El Sargento, 27-II-1984, R. Riosmena (FBCS 1962), 15-III-1994, R. Uripiti y R. Rivera (FBCS 627), Cabo Pulmo, 25-IV-1990, R. Riosmena (FBCS 2155), 6-III-1994, R. Riosmena (FBCS 1120) (Anaya y Riosmena-Rodríguez, 1996: 863; Mateo-Cid et al. 2000:10); Cabeza Ballena, 11-III-1949, E. Y. Dawson (LAM 501271). .SONORA: Puerto Peñasco (Dawson, 1966: 18 como $A$. zonata y $A$. drouetii); Bahía Kino, 16-X1983, L. E. Mateo-Cid y C. Flores (ENCB 8931) (MendozaGonzález y Mateo-Cid, 1986: 422); Isla Turner, 18-VII1940, E. Y. Dawson (LAM 55175); Bahía de Guaymas 23-I-1940, E. Y. Dawson (LAM 55145 y UC 974730) (Dawson, 1944: 276 como A. zonata); Punta Colorada, 13II-1946, E. Y. Dawson (LAM 4274), 22-XI-1946, E. Y. Dawson (LAM 55142); Bahía de Bacochibampo, Playa Miramar, 16-V-1946, E. Y. Dawson (LAM 4795); 26-X1991, C. Galicia (ENCB 8929); Playa San Carlos (Bahía San Francisco), 27-X-1991, C. Galicia (ENCB 8928). 
SINALOA: Mazatlán, 27-VII-1987, A.C. MendozaGonzález y L. E. Mateo-Cid (ENCB 8939) (MendozaGonzález et al. 1994: 106). NAYARIT: Isla María Madre (Dawson, 1961: 421 como A. zonata); Bahía Chacala (Mateo-Cid y Mendoza-González, 1992: 20); Lo de Marcos, 9-XI-1991, L. E. Mateo-Cid y C. Galicia (ENCB 12221) (Mateo-Cid y Mendoza-González, 1992: 20); Playa Guayabitos, 6-VII-1985, A.C. Mendoza-González y L. E. Mateo-Cid y B. López (ENCB 8937) (Mateo-Cid y Mendoza-González, 1992: 20); Punta de Mita, 22-VI1989, L. E. Mateo-Cid y B. López (ENCB 8934) (MateoCid y Mendoza-González, 1992: 20; Serviere-Zaragoza et al. 1993: 482). JALISCO: Puerto Vallarta (Playa Los Muertos-Conchas Chinas), 21-VII-1987, A. C. MendozaGonzález y L. E. Mateo-Cid (ENCB 8955), 21-VI-1989, L. E. Mateo-Cid y B. López (ENCB 8951) (MendozaGonzález y Mateo-Cid, 1992: 18 como A. zonata); Yelapa, 5-VII-1985, A.C. Mendoza-González y L. E. Mateo-Cid (ENCB 8957), Chamela, 15-VIII-1986, A. Quintanar (UAMIZ 372), 6-X-1991, L. Huerta y A. C. MendozaGonzález (ENCB 8952), 11-VII-1992, L. E. Mateo-Cid y C. Galicia (ENCB 8961), Tenacatita 12-VII-1992, L. E. Mateo-Cid y C. Galicia (ENCB 8962), Careyes, 8-X-1991, A.C. Mendoza-González y L. E. Mateo-Cid (ENCB 9850), 13-VII-1992, L. E. Mateo-Cid y C. Galicia (ENCB 8958), La Manzanilla, 14-VII-1992, L. E. Mateo-Cid y C. Galicia (ENCB 8948), Bahía de Cuastecomate 9-X-1991, A.C. Mendoza-González y L. E. Mateo-Cid (ENCB 8949), 15VII-1992, L. E. Mateo-Cid y C. Galicia (ENCB 8956), Melaque, 3-X-1991, A. C. Mendoza-González y C. Galicia (ENCB 8953), 10-VII-1992, L. E. Mateo-Cid y C. Galicia (ENCB 8960). COLIMA: Isla Socorro, 21-III-1967, L. Huerta-Múzquiz y A. M. Garza B. (ENCB 1127) (HuertaMúzquiz y Garza Barrientos, 1975: 14 como A. drouetii); Manzanillo(Playa Santiago), 16-XII-1985, A. C. MendozaGonzález y L. E. Mateo-Cid (ENCB 8938) (Mateo-Cid y Mendoza-González, 1991: 19 como A. drouetii). MICHOACÁN: San Telmo (Dreckmann et al. 1990: 37 como A. mexicana y A. franciscana); Faro de Bucerías o El Faro, 19-IX-1986, A. Sentíes (UAMIZ 70), 23-V-1988, A. Sentíes (UAMIZ 27), 10-XII-1988, G. Cevallos (ENCB 8945), (Stout y Dreckmann, 1993: 12), Maruata, 16-V1986, A. C. Mendoza-González y L. E. Mateo-Cid (ENCB 8944), 9-IX-1987, A. C. Mendoza-González y L. E. MateoCid (ENCB 8946), 9-XII-1987, A. Sentíes y F. F. Pedroche (UAMIZ 264), 10-XII-1988, G. Cevallos (ENCB 8942); Caletilla, 3-III-2007, A. C. Mendoza-González y L.E. Mateo Cid (ENCB s/n). GUERRERO: Morro de Petatlán (Dawson, 1961: 421 como A. mexicana); Isla Ixtapa o Grande (Playa Coral), 10-IV-1959, E. Y. Dawson (US 20954), 7-XI-1996, L. E. Mateo-Cid y C. MendozaGonzález (ENCB 12217) (Mendoza-González y Mateo-
Cid, 1998: 19); Playa Las Gatas, 3-IX-1995, L. E. y B. López, (ENCB 8936), 8-XI-1996, L. E. Mateo-Cid y A. C. Mendoza-González (ENCB 12216), (Mendoza-González y Mateo-Cid, 1998: 19), Barra de Potosí, 6-XI-1996, L. E. Mateo-Cid y A. C. Mendoza-González (ENCB 12231), Bahía Petatlán, 2-III-1934, W. R. Taylor (LAM 501338) (Taylor, 1945: 188 como A. peninsularis); Acapulco, 3-II1947, E. Y. Dawson (LAM 501274) (Dawson, 1953: 150 como A. franciscana var. robusta; Mendoza-González y Mateo-Cid, 1998: 19). OAXACA: Morro de Cerro Hermoso, 23-II-1996, A. C. Mendoza-González y L. E. Mateo-Cid (ENCB 12218) (Mateo-Cid y MendozaGonzález, 2001:19); Puerto Angelito 7-XII-1985, Celia Flores y O. Holguín (ENCB 8970); Puerto Escondido, 30IX-1992, A. C. Mendoza-González y L. E. Mateo-Cid (ENCB 8983) (Mateo-Cid y Mendoza-González, 2001:19), 7-VIII-1993, L. E. Mateo-Cid y A. C. Mendoza-González (ENCB 8981), 25-II-1996, L. E. Mateo-Cid y A. C. Mendoza-González (ENCB 12220) (Mendoza-González y Mateo-Cid, 1998: 19); Playa Agua Blanca, 27-II-1996, A. C. Mendoza-González y L. E. Mateo-Cid (ENCB 8984) (Mateo-Cid y Mendoza-González, 2001:19); Santa Elena, 9-XII-1994, L. E. Mateo-Cid y A. C. Mendoza-González (ENCB 8982) (Mateo-Cid y Mendoza-González, 2001:19), 22-II-1996, L. E. Mateo-Cid y A. C. Mendoza-González (ENCB 12219); Puerto Ángel, 29-IX-1992, A. C. MendozaGonzález y L. E. Mateo-Cid (ENCB 8964), 8-XII-1994, A. C. Mendoza-González y L. E. Mateo-Cid (ENCB 8974) (León-Tejera, 1996: 48, Mendoza-González y Mateo-Cid, 1998: 19, Mateo-Cid y Mendoza-González, 2001:19); Bahía Santa Cruz, 26-IX-1992, A. C. Mendoza-González y L. E. Mateo-Cid (ENCB 8973); Bahía Tangolunda, 28II-1934, W. R. Taylor (LAM 1515), 28-IX-1992, A. C. Mendoza-González y L. E. Mateo-Cid (ENCB 8972), 8VIII-1993, A. C. Mendoza-González y L. E. Mateo-Cid (ENCB 8975) (Mateo-Cid y Mendoza-González, 2001:19); Playa La Entrega, 25-IX-1992, A. C. Mendoza-González y L. E. Mateo-Cid (ENCB 8994); La Ventosa, 6-VII-1992, L. E. Mateo-Cid y A. C. Mendoza-González (ENCB 8980) (Mateo-Cid y Mendoza-González, 2001:19), L. HuertaMúzquiz (ENCB 1843); Punta Conejo, 7-VIII-1992, A. C. Mendoza-González y L. E. Mateo-Cid (ENCB 8969) (Mateo-Cid y Mendoza-González, 2001:19); Salina Cruz, 9-I-1947, E. Y. Dawson (LAM 51176) (Dawson, 1961: 421 como A. mexicana); 9-VIII-1969, L. Huerta-Múzquiz (ENCB 8966), 17-XI-1989, K. Dreckmann (UAMIZ 183 y 166); 8-VIII-1992, A. C. Mendoza-González y L. E. Mateo-Cid (ENCB 8968) (Huerta-Múzquiz y Tirado, 1970: como A. mexicana; Galindo-Villegas, et al., 1997: 4; Mendoza-González y Mateo-Cid, 1998: 19, Mateo-Cid y Mendoza-González, 2001:19). YUCATÁN: Isla Desertora, 15-X-1985, Laura Huerta y L. E. Mateo-Cid (ENCB 8940). 
QUINTANA ROO: Puerto Morelos (Huerta-Múzquiz et al., 1987: 45) Akumal (Aguilar-Rosas et al., 1998: 24); Xcalak (Huerta-Múzquiz et al., 1987: 45); Banco Chinchorro (Huerta-Múzquiz et al., 1987: 45); Parte este de Isla Mujeres, 14-VIII-1997, Richard B. Searles (ENCB 15624).

Hábitat. Sobre rocas, intermareal expuesto y en el submareal hasta $10 \mathrm{~m}$ profundidad.

Distribución mundial y en México. Esta especie se encuentra ampliamente distribuida, se tienen registros de ella en los siguientes países y regiones: Grecia, Francia, Italia, Malta, España, Turquía, islas Azores, islas Canarias. En América del Norte se le conoce en Florida, Carolina del Norte, Carolina del Sur. En el Caribe: Cuba. América del Sur: Brasil, Uruguay, Chile y Venezuela. África: Angola, Congo, Egipto, Guinea Ecuatorial, Gambia, Ghana, Liberia, Sudáfrica, Tanzania, Marruecos, Túnez, Senegal. Asia: Omán, Filipinas, República Árabe de Siria, Indonesia, Vietnam y en el Pacífico se ha registrado en Fiji, Hawaii, Panamá y California (Guiry y Guiry, 2006).

Por lo que respecta a México, $A$. beauvoisii es la especie que presenta la distribución más amplia en la costa del Pacífico, es muy rara en el Caribe mexicano y es dato interesante el que aún no ha sido registrada en el Golfo de México (Ortega et al., 2001).

Considerando las campañas de recolecta en 18 localidades del litoral del Pacífico y Caribe mexicano, así como los ejemplares de herbario, se examinaron 256 especímenes de $A$. beauvoisii. Se encontró que esta especie se distribuye en la costa del Pacífico desde iIslas Coronados, Baja California hasta La Ventosa, Oaxaca, siendo muy frecuente en el golfo de California y en los estados de Jalisco, Guerrero y Oaxaca, mientras que en la costa del Atlántico su distribución se encuentra restringida a los arrecifes coralinos de Yucatán y Quintana Roo.

Variación morfológica. Una de las características más notorias de $A$. beauvoisii es su gran polimorfismo, lo que ha generado que haya sido determinada y depositada en herbarios bajo otros nombres, sobre todo los especímenes del golfo de California, lo que también han observado Norris y Johansen (1981) y Riosmena-Rodríguez y Siqueiros-Beltrones (1996) en poblaciones del golfo de California. Esta especie está presente en áreas tropicales y subtropicales; por ejemplo, en Brasil. Moura y Beauclair (2005) indican que los especímenes de A. beauvoisii son altamente polimórficos y aparentemente su morfología está relacionada con el ambiente donde habitan; además mencionan que en lugares protegidos los talos son erectos y laxos, subcilíndricos a comprimidos, mientras que en las zona expuestas los talos son postrados, agregados y comprimidos. Como puede observarse en las figuras 34, 35 y 36, los ejemplares de $A$. beauvoisii presentes en el Pacífico de México difieren de lo descrito por Moura y Beauclair (2005), ya que los talos erectos y laxos se encuentran en regiones expuestas, como sucede en Oaxaca y Michoacán (Pacifico tropical mexicano); en contraste, en el Golfo de California, los talos son pequeños postrados y agregados (Figs. 32,33); aparentemente la morfología externa de los talos está relacionada con el tipo de ambiente en el que se desarrollan.

Las características morfológicas, anatómicas y de reproducción encontradas en ejemplares mexicanos de $A$. beauvoisii se señalan en los cuadros 1 y 2 , donde se puede observar que existen diferencias significativas entre las poblaciones de $A$. beauvoisii que habitan en el golfo de California y en el Pacífico tropical de México. Por otro lado, en las figuras 32 a 44 también se observa que las diferencias de hábito entre el bisporofito y el tetrasporofito son notorias. Los datos presentados por Norris y Johansen (1981) y Riosmena-Rodríguez y Siqueiros-Beltrones (1996) incluyen tanto los talos bisporangiales como tetrasporangiales, por lo que no es posible establecer comparaciones con nuestros resultados. En las figuras 19 a 44 se observa la notable variación morfológica tanto de los bisporofitos como de los tetrasporofitos y en los cuadros 1 y 2 se indican los caracteres utilizados para conocer la variación morfológica de $A$. beauvoisii, lo cual nos muestra que los caracteres internos de los talos, tales como número de capas en la corteza, alternancia de células largas y cortas de la médula y sus dimensiones, así como el número de capas de la genícula son constantes y las diferencias mayores se encuentran en la talla de los talos, la longitud y forma de las intergenículas y el diámetro de las cámaras, ya sean bisporangiales o tetrasporangiales. Asimismo se observó que existen diferencias notables entre el aspecto morfológico de los esporofitos con respecto de los gametofitos (Figs. 1-8).

Distribución latitudinal de las fases reproductivas .El análisis de la información de herbarios y recolectas realizadas, confirmó la presencia de $A$. beauvoisii en 101 localidades a lo largo de la costa de México; además, reveló una marcada variación latitudinal de los talos tetrasporangiales, bisporangiales y gametangiales (carposporangiales y espermatangiales). Los talos tetrasporangiales muestran una amplia distribución geográfica al estar presentes en 5 de las 6 regiones geográficas consideradas en este estudio. Las plantas bisporangiales se encuentran bien representadas a todo lo largo de la costa del Pacífico de México. Por otro lado, las escasas plantas gametangiales se presentan en los estados de Jalisco y Oaxaca, en ambientes protegidos de aguas más cálidas, como playa Careyes y bahía Tangolunda.

Los talos de A. beauvoisii son perennes y crecen sobre sustrato rocoso, epífitos o epizoicos. La talla de los talos 

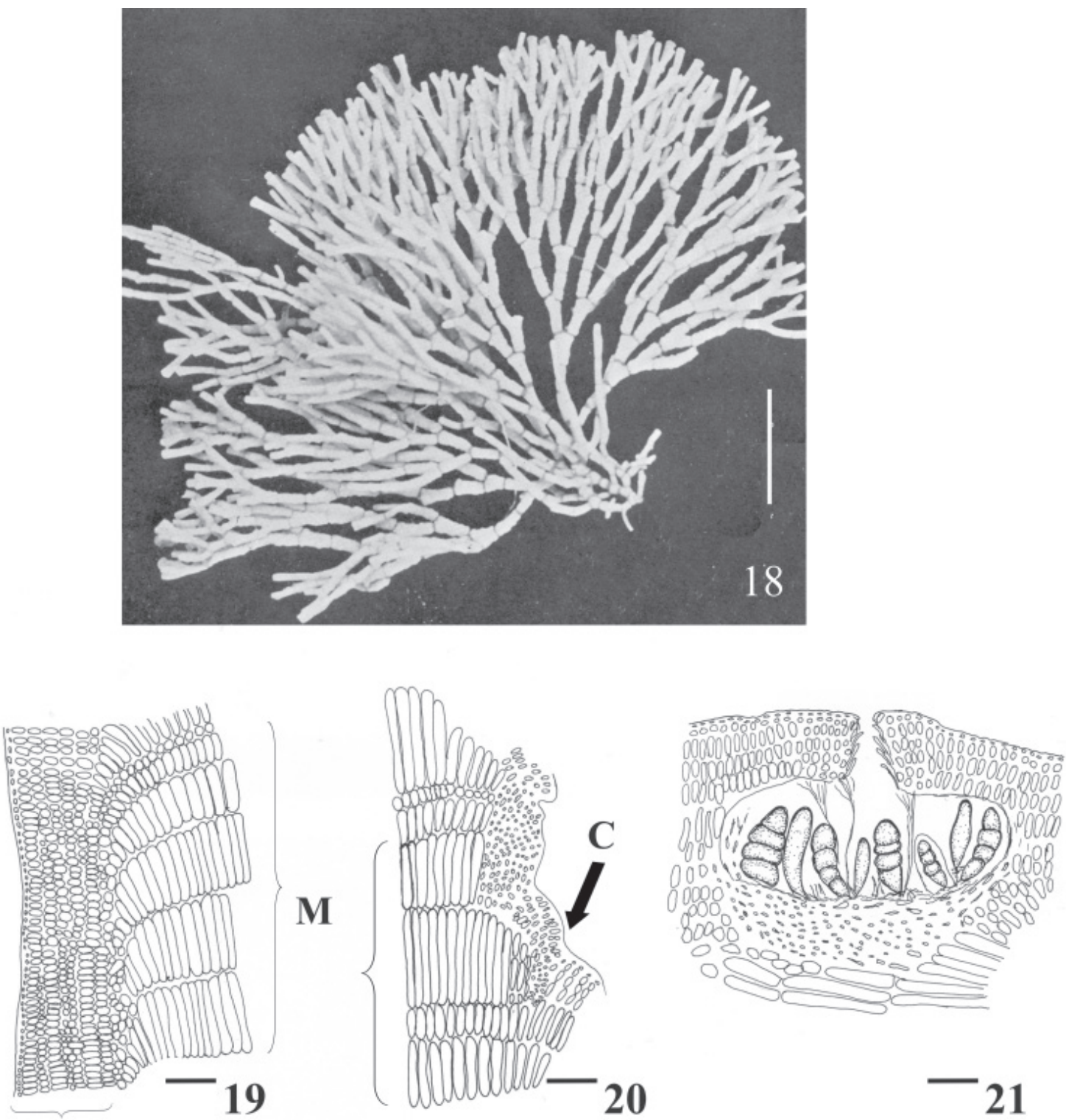

C

Figuras 18- 21. Amphiroa mexicana. 18. Hábito de la planta tetrasporangial (LAM 3826). Bahía Petatlán, Guerrero, 17 de marzo 1933. 19. Esquema en cámara clara de un corte longitudinal de la intergenícula del ejemplar TIPO, donde se observa la corteza (C) y la médula (M). 20. Esquema en cámara clara de un corte longitudinal de la genícula del ejemplar TIPO, donde se observa la corteza (C) y la médula (M). 21. Detalle del conceptáculo tetrasporangial del ejemplar TIPO. Esquema en cámara clara. Escalas, 18: $1 \mathrm{~cm}$, 19-21: $60 \mu \mathrm{m}$.

analizados para la costa del Pacífico tropical de México es de 2-10 cm; mayores que los registrados previamente por Dawson (1953) para la misma región. Los bisporangiales dominan a lo largo de la costa occidental de la península de Baja California, lo que concuerda con el patrón de distribución que mencionan Tyrrell y Johansen (1995) para poblaciones de Lithothrix aspergillum establecidas a lo largo de la costa del Pacífico de Baja California, ya que éstas se mantienen sólo a través de talos bisporangiales o vegetativos, estando las plantas tetrasporangiales y gametangiales ausentes debido a la presencia de aguas frías producidas por el fenómeno de surgencias a lo largo de la costa, cuya temperatura superficial se encuentra por debajo de los $14{ }^{\circ} \mathrm{C}$ (Aguilar-Rosas et al., 2007). Por otro lado, la 
Cuadro 1. Caracteres de la fase tetrasporangial de Amphiroa beauvoisii en las regiones de la costa del Pacífico y Caribe de México

\begin{tabular}{|c|c|c|c|c|c|c|c|c|}
\hline Región & $\begin{array}{l}\text { Talla } \\
(\mathrm{cm})\end{array}$ & $\begin{array}{l}\text { Forma/long } \\
\text { lancho } \\
\text { intergenícula } \\
\text { media }(\mathrm{mm})\end{array}$ & $\begin{array}{l}\text { Formal } \\
\text { long/ ancho } \\
\text { intergenícula } \\
\text { apical }(\mu \mathrm{m})\end{array}$ & $\begin{array}{l}\text { Long/ } \\
\text { diam } \\
\text { células } \\
\text { medulares } \\
(\mu \mathrm{m})\end{array}$ & $\begin{array}{l}\text { Número } \\
\text { capas } \\
\text { corteza }\end{array}$ & $\begin{array}{l}\text { Número } \\
\text { capas } \\
\text { genícula }\end{array}$ & $\begin{array}{l}\text { Diam/alto } \\
\text { cámaras } \\
\text { tetraspo- } \\
\text { rangiales } \\
(\mu \mathrm{m})\end{array}$ & $\begin{array}{l}\text { Long/diam } \\
\text { tetraspo- } \\
\text { rangio } \\
(\mu \mathrm{m})\end{array}$ \\
\hline $\begin{array}{l}\text { Golfo } \\
\text { California } \\
\text { (B. C., } \\
\text { Sonora) }\end{array}$ & $1.5-5.5$ & $\begin{array}{c}\text { Comprimida } \\
2-4 / 0.4-1.2\end{array}$ & $\begin{array}{l}\text { Cilíndrica- } \\
\text { comprimida } \\
\text { 2-6/0.5-0.8 }\end{array}$ & $\begin{array}{c}48-112 / \\
8-12.5\end{array}$ & $3-7$ & $\begin{array}{l}4-6 \text { con } \\
\text { o sin } \\
\text { corticación }\end{array}$ & $\begin{array}{l}148-260 / \\
95-115\end{array}$ & $\begin{array}{c}40-68 / 25- \\
35\end{array}$ \\
\hline $\begin{array}{l}\text { Golfo } \\
\text { California } \\
\text { (B. C. S., } \\
\text { Sinaloa) }\end{array}$ & $1.5-5.0$ & $\begin{array}{l}\text { Comprimida } \\
1-3.5 / 0.5-1.0\end{array}$ & $\begin{array}{l}\text { Cilíndrica- } \\
\text { comprimida } \\
1-5 / 0.3-0.5\end{array}$ & $\begin{array}{c}62.7-81.8 / \\
8.5-12\end{array}$ & $3-8$ & $\begin{array}{l}4-6 \text { con } \\
\text { o sin } \\
\text { corticación }\end{array}$ & $\begin{array}{l}205-255 / \\
120-150\end{array}$ & $\begin{array}{c}60- \\
66 / 22.8- \\
30\end{array}$ \\
\hline $\begin{array}{l}\text { Pacífico } \\
\text { (Tijuana, B. } \\
\text { Magdalena }\end{array}$ & ------- & -------------- & -------------- & ------------ & -------- & ------------- & ------------- & ------ \\
\hline $\begin{array}{l}\text { Pacífico } \\
\text { (Punta } \\
\text { Conejo, } \\
\text { Cabo San } \\
\text { Lucas) }\end{array}$ & $3.5-6.5$ & $\begin{array}{c}\text { Comprimida } \\
2-4 / 1-1.2\end{array}$ & $\begin{array}{c}\text { Comprimida } \\
4-5 / 0.6-0.8\end{array}$ & $\begin{array}{c}74-92 / \\
7-8\end{array}$ & $5-6$ & $\begin{array}{l}3-4 \\
\text { corticada }\end{array}$ & $\begin{array}{c}148-200 / \\
74-94\end{array}$ & $\begin{array}{c}63-67 / 26- \\
30\end{array}$ \\
\hline $\begin{array}{l}\text { Pacífico } \\
\text { tropical } \\
\text { mexicano** }\end{array}$ & $2-10$ & $\begin{array}{l}\text { Cilíndrica- } \\
\text { comprimida } \\
1-7 / 0.6-2.0\end{array}$ & $\begin{array}{l}\text { Comprimida- } \\
\text { aplanada } \\
2-5 / 0.3-1.5\end{array}$ & $\begin{array}{c}66-118 / \\
7-12\end{array}$ & $2-9$ & $\begin{array}{l}3-10 \text { con } \\
\text { o sin } \\
\text { corticación }\end{array}$ & $\begin{array}{c}214-300 / \\
90-135\end{array}$ & $\begin{array}{c}45-92 / 26- \\
40\end{array}$ \\
\hline Caribe & $2.5-3.0$ & $\begin{array}{c}\text { Comprimida } \\
2-4 / 1.0-1.2\end{array}$ & $\begin{array}{c}\text { Comprimida } \\
2-6 / 0.7-0.9\end{array}$ & $\begin{array}{c}62.9-111 / \\
11-12\end{array}$ & $3-5$ & $\begin{array}{l}2-3 \\
\text { corticada }\end{array}$ & $\begin{array}{c}150-180 / \\
105-110\end{array}$ & $\begin{array}{c}35- \\
40 / 18.5- \\
20\end{array}$ \\
\hline $\begin{array}{l}\text { Amphiroa } \\
\text { mexicana } \\
\text { (Tipo) }\end{array}$ & $3-4.5$ & $\begin{array}{l}\text { Cilíndrica- } \\
\text { comprimida } \\
2-4 / 0.8-1.2\end{array}$ & $\begin{array}{c}\text { Cilíndrica- } \\
\text { subcilíndrica } \\
1.5-3 / 0.5-0.6\end{array}$ & $\begin{array}{c}62.9-111 / \\
11-12\end{array}$ & $6-8$ & $\begin{array}{l}5-7 \\
\text { corticada }\end{array}$ & $\begin{array}{l}185-222 / \\
111-121\end{array}$ & $\begin{array}{c}71-74 / 31- \\
37\end{array}$ \\
\hline
\end{tabular}

* Todos los ejemplares tienen la intergenícula basal cilíndrica.

** Incluye los estados de Nayarit, Jalisco, Colima, Michoacán, Guerrero y Oaxaca.

fase bisporangial de $A$. beauvoisii es dominante en el golfo de California, donde el $65 \%$ de los especímenes revisados son bisporangiales y sólo el $35 \%$ son tetrasporangiales. En contraste, los talos tetrasporangiales muestran una amplia distribución geográfica tanto en el golfo de California como en el Pacífico tropical de México. En esta última región representaron el $60 \%$ y los bisporangiales el $40 \%$. Posiblemente las áreas costeras influenciadas por aguas frías de surgencias, con temperaturas menores de $14{ }^{\circ} \mathrm{C}$ (Dawson, 1951; 1954) y la presencia de bahías protegidas con variaciones de $16-30{ }^{\circ} \mathrm{C}$ de temperatura anual (GómezGutiérrez et al., 2001) son las que determinan el patrón latitudinal de las fases reproductivas en el Pacífico de México.
Dependiendo de la fase reproductiva, las plantas muestran una marcada variación morfológica (Figs. 19-44). En general, los talos tetrasporangiales son más grandes (10 $\mathrm{cm}$.), muy ramificados y arborescentes, el diámetro de las cámaras (Fig. 28) es menor que el de las bisporangiales, en comparación a los talos bisporangiales que son pequeños (6 cm.), compactos, más ramificados y los diámetros de las cámaras bisporangiales más grandes (Fig. 29). Los talos gametangiales (carposporangiales y espermatangiales) son más pequeños, y con numerosas ramas laterales (Figs. 1,2). Al comparar los caracteres morfométricos de los conceptáculos para los talos tetrasporangiales, bisporangiales, carposporangiales y espermatangiales, descritos por Dawson (1953), Norris y Johansen (1981) 
Cuadro 2. Caracteres de la fase bisporangial de Amphiroa beauvoisii en las regiones de la costa del Pacífico y Caribe de México

\begin{tabular}{|c|c|c|c|c|c|c|c|c|}
\hline Región & $\begin{array}{l}\text { Talla } \\
(\mathrm{cm})\end{array}$ & $\begin{array}{l}\text { Forma/long } \\
\text { lancho } \\
\text { intergenicula } \\
\text { media (mm) }\end{array}$ & $\begin{array}{l}\text { Forma/long } \\
\text { lancho } \\
\text { intergenicula } \\
\text { apical }\end{array}$ & $\begin{array}{l}\text { Long/ } \\
\text { diam } \\
\text { células } \\
\text { medulares } \\
(\mu \mathrm{m})\end{array}$ & $\begin{array}{l}\text { Núm. } \\
\text { capas } \\
\text { corteza }\end{array}$ & $\begin{array}{l}\text { Núm. capas } \\
\text { genícula }\end{array}$ & $\begin{array}{l}\text { Diam/alto } \\
\text { cámara } \\
\text { bisporangial } \\
(\mu \mathrm{m})\end{array}$ & $\begin{array}{l}\text { Long/diam } \\
\text { bisporan- } \\
\text { gio }(\mu \mathrm{m})\end{array}$ \\
\hline $\begin{array}{l}\text { Golfo } \\
\text { California } \\
\text { (B.C., } \\
\text { Sonora) }\end{array}$ & $2.2-5$ & $\begin{array}{l}\text { Cilíndrica- } \\
\text { comprimida } \\
2-5 / 0.8-1.2\end{array}$ & $\begin{array}{l}\text { Cilíndrica- } \\
\text { comprimida } \\
2-6 / 0.5-1.0\end{array}$ & $\begin{array}{l}62.9-125 / \\
7.4-11.5\end{array}$ & $2-5$ & $\begin{array}{l}3-5 \text { con o sin } \\
\text { corticación }\end{array}$ & $\begin{array}{l}240-320 / \\
105-135\end{array}$ & $\begin{array}{l}40-67 / 22- \\
40\end{array}$ \\
\hline $\begin{array}{l}\text { Golfo } \\
\text { California } \\
\text { (B.C. Sur, } \\
\text { Sinaloa) }\end{array}$ & $2-3.2$ & $\begin{array}{l}\text { Comprimida } \\
2-4 / 1.0-1.2\end{array}$ & $\begin{array}{l}\text { Comprimida } \\
1-2 / 0.3-0.5\end{array}$ & $\begin{array}{l}62-92 / \\
9-11\end{array}$ & $4-6$ & $\begin{array}{l}4-6 \\
\text { parcialmente } \\
\text { corticada } \\
\text { o no }\end{array}$ & $\begin{array}{l}240-300 / \\
70-105\end{array}$ & $\begin{array}{l}45-62 / 22- \\
31\end{array}$ \\
\hline $\begin{array}{l}\text { Pacífico } \\
\text { (Tijuana, B. } \\
\text { Magdalena }\end{array}$ & $3.5-6$ & $\begin{array}{l}\text { Comprimida } \\
3-7 / 1.0-2.0\end{array}$ & $\begin{array}{l}\text { Cilíndrica- } \\
\text { comprimida } \\
2-7.5 / 0.6-1.0\end{array}$ & $\begin{array}{l}65-148 / \\
7.4-9.2\end{array}$ & $4-8$ & $\begin{array}{l}4-6 \\
\text { corticada }\end{array}$ & $\begin{array}{l}375-480 / \\
120-150\end{array}$ & $\begin{array}{l}55-70 / 23- \\
29.6\end{array}$ \\
\hline $\begin{array}{l}\text { Pacífico } \\
\text { (Punta } \\
\text { Conejo, } \\
\text { Cabo San } \\
\text { Lucas) }\end{array}$ & $1-4$ & $\begin{array}{l}\text { Comprimida } \\
3-6 / 1.0-1.5\end{array}$ & $\begin{array}{l}\text { Cilíndrica } \\
\text { 7-11/0.5-0.6 }\end{array}$ & $\begin{array}{l}74-111 / \\
10-11\end{array}$ & $2-6$ & $\begin{array}{l}4-6 \\
\text { corticada }\end{array}$ & $\begin{array}{l}300-380 / \\
120-135\end{array}$ & $\begin{array}{l}44-80 / 30- \\
46\end{array}$ \\
\hline $\begin{array}{l}\text { Pacífico } \\
\text { tropical } \\
\text { mexicano** }\end{array}$ & $1.2-6$ & $\begin{array}{l}\text { Comprimida } \\
1-6 / 0.5-0.6\end{array}$ & $\begin{array}{l}\text { Cilíndrica- } \\
\text { comprimida } \\
2-5 / 0.5-0.7\end{array}$ & $\begin{array}{l}64-92 / \\
7.4-13\end{array}$ & 4-6 & $\begin{array}{l}4-6 \\
\text { parcialmente } \\
\text { corticada }\end{array}$ & $\begin{array}{l}240-330 / \\
105-115\end{array}$ & $\begin{array}{l}52-60 / 25- \\
29\end{array}$ \\
\hline Caribe & ------ & --------------- & ---------------- & ------------ & --------- & ---------------- & --------------- & ----------- \\
\hline
\end{tabular}

* Todos los ejemplares tienen la intergenícula basal cilíndrica.

** Incluye los estados de Nayarit, Jalisco, Colima, Michoacán, Guerrero y Oaxaca.

y Riosmena-Rodríguez y Siqueiros Beltrones (1996), con los determinados en el presente estudio, se encontraron algunas diferencias. Nuestros datos indican que las cámaras tetrasporangiales y bisporangiales son más grandes; por otro lado, las cámaras carposporangiales y espermatangiales son más anchas y de menor altura (Cuadros 1 y 2). Las dimensiones de los tetrasporangios y bisporangios corresponden a los intervalos determinados por los autores citados anteriormente.

\section{Análisis taxonómico}

Muchos especímenes de A. beauvoisii fueron colectados en el golfo de California y en el Pacifico de México y se citaron con otros nombres como A. zonata, A. crosslandii, A. drouetii y A. linearis, entre otros
(Taylor 1945; Dawson 1953; González-González et al. 1996). Estudios posteriores demostraron que la especie es altamente variable, por lo que fue necesario incluir estas especies dentro de A. beauvoisii (Norris y Johansen 1981, Riosmena-Rodríguez y Siqueiros-Beltrones 1996). En el caso de Amphiroa mexicana W. Taylor, se revisó el tipo depositado en LAM (Figs. 18-21) y se encontró que presenta características similares a los especímenes de $A$. beauvoisii recolectados en Sinaloa, Baja California Sur, Guerrero, Oaxaca y Michoacán (Figs. 22-29, 3537; Cuadro 1), por lo que consideramos que es válido considerar a A. mexicana como sinónimo taxonómico de A. beauvoisii.

Las características morfológicas distintivas que permiten separar a $A$. beauvoisii de otras especies de Amphiroa presentes en México, como A. rigida J.V. Lamouroux y $A$. van-bossea Lemoine, son la forma de 

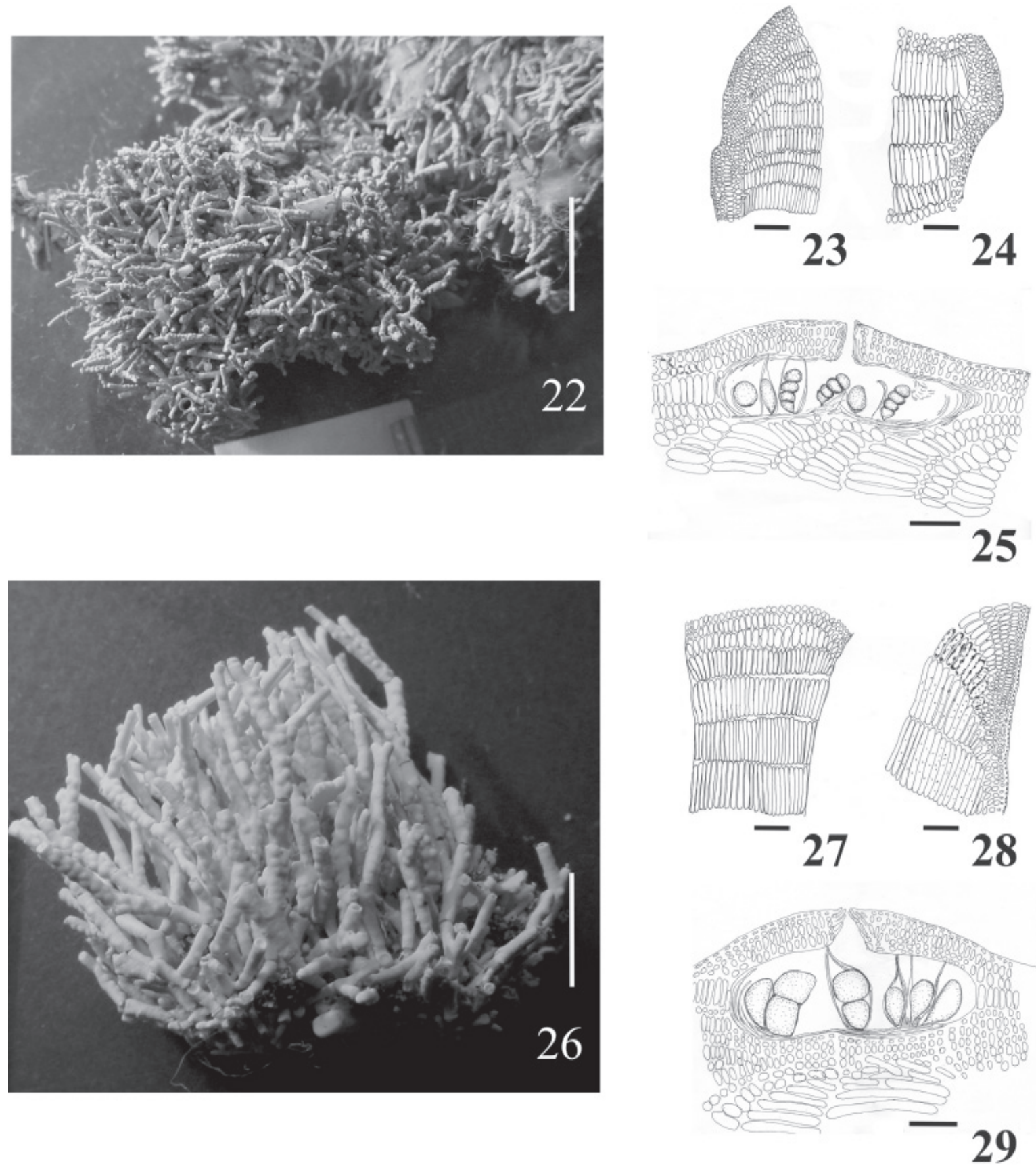

Figuras 22 - 29. Amphiroa beauvoisii. 22. Hábito de la planta tetrasporangial (ENCB 8939). Mazatlán, Sinaloa, 27 de julio 1987. 23. Esquema en cámara clara de un corte longitudinal de la intergenícula (ENCB 8939). 24. Esquema en cámara clara de un corte longitudinal de la genícula (ENCB 8939). 25. Detalle del conceptáculo tetrasporangial (ENCB 8939). 26. Hábito de la planta bisporangial (ENCB 7990). Todos Santos, BCS, 17 de mayo 1989. 27. Esquema en cámara clara de un corte longitudinal de la genícula (ENCB 7990). 28. Esquema en cámara clara de un corte longitudinal de la intergenícula (ENCB 7990). 29. Detalle del conceptáculo bisporangial (ENCB 7990). Escalas, 22 y 26: $1 \mathrm{~cm}, 23-25$ y 27-29: $60 \mu \mathrm{m}$.

las intergenículas, que en estas especies son cilíndricas o subcilíndricas y que en el caso de $A$. van-bossea rebasan los $2 \mathrm{~mm}$ de diámetro, así como el número de capas de células largas que alternan con una capa de células cortas, el número de capas de la genícula que en $A$. rigida generalmente son 2 y en $A$. van-bossea de 5-10, mientras que el diámetro de las cámaras tetrasporangiales de $A$. rigida no sobrepasa $175 \mu \mathrm{m}$. 


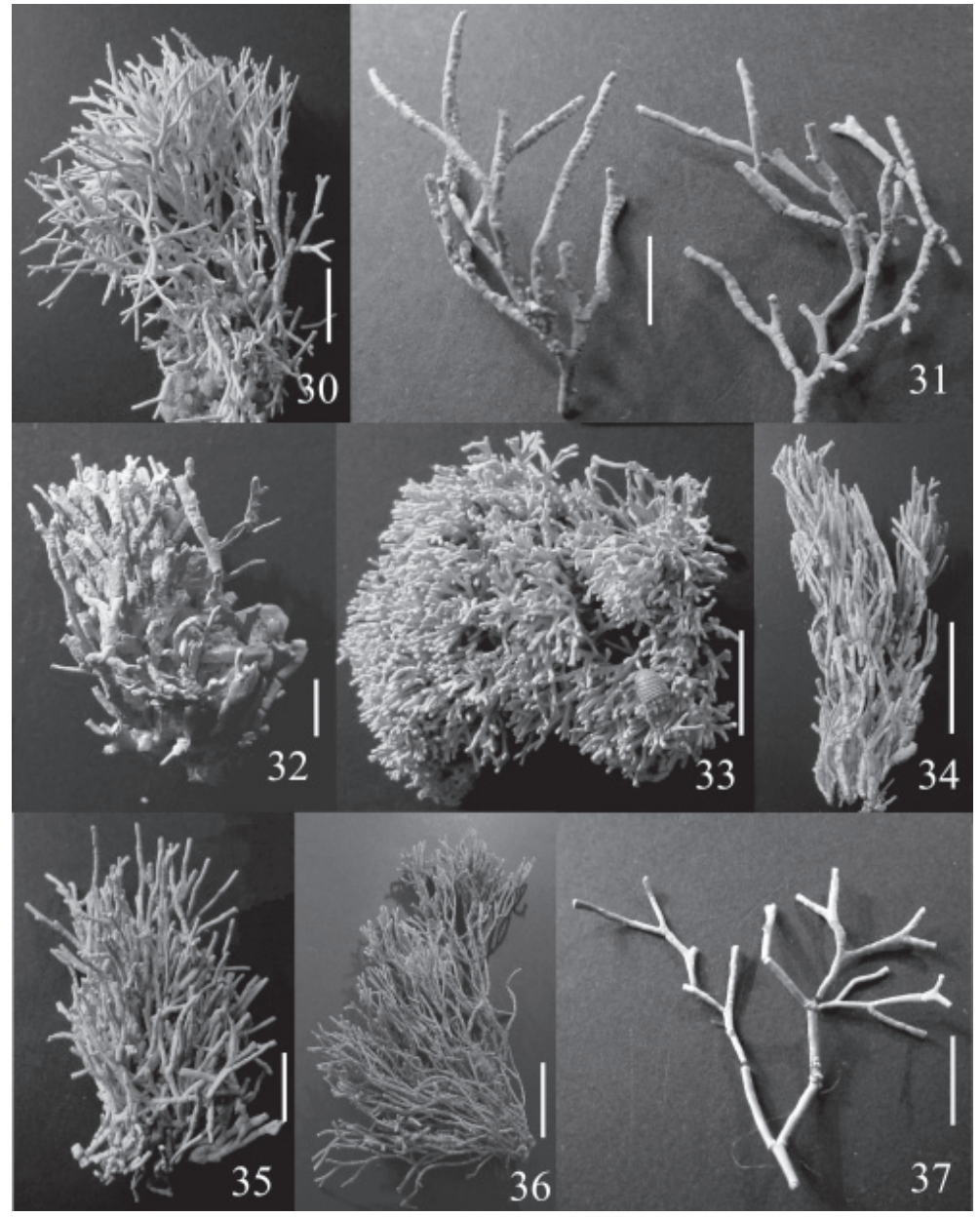

Figuras 30-37. Amphiroa beauvoisii. Variación morfológica de talos tetrasporangiales. 30. Ejemplar de Playa El Coloradito, B.C. 26 de julio de 1996 (ENCB 8900). 31. Ejemplar de Puertecitos, B.C. 4 de febrero de 1995 (ENCB 7994). 32. Ejemplar de Playa Norte, Mazatlán, Sinaloa. 26 de julio de 1984 (ENCB 12473). 33. Ejemplar de Las Conchas, Bahía Concepción, B.C.S. 23 de mayo de 1990 (ENCB 7999). 34. Ejemplar de Todos Santos, B.C.S. 17 de octubre de 1989 (ENCB 7995. Escala: $2 \mathrm{~cm}$ ). 35. Ejemplar de Careyes, Jalisco. 8 de octubre de 1991 (ENCB 8950). 36. Ejemplar de Caletilla, Michoacán, 8 de marzo del 2007 (ENCB $\mathrm{s} / \mathrm{n})$. 37. Ejemplar de la parte este de Isla Mujeres, Quintana Roo, 14 de agosto de 1997 (ENCB 15624). Escalas, 30, 31, 35 y 37: $1 \mathrm{~cm}, 32: 5 \mathrm{~mm}, 33: 6 \mathrm{~mm}, 34$ y $36: 2$ $\mathrm{cm}$.

\section{Consideraciones finales}

A partir de los resultados obtenidos en este trabajo, consideramos que es necesario realizar estudios ecológicos y poblacionales de $A$. beauvoisii con el fin de precisar la relación entre la morfología de los talos y los factores ambientales que determinan dichas variaciones. Asimismo, es importante esclarecer la dominancia de las distintas fases reproductivas. Fue evidente la escasez de registros y recolectas de especímenes de esta especie en el Caribe y la Costa del Atlántico de México, por lo que es necesario realizar campañas de muestreo que permitan obtener mayor información sobre la presencia de $A$. beauvoisii en esta región.

\section{Agradecimientos}

Se agradece a la Escuela Nacional de Ciencias Biológicas del Instituto Politécnico Nacional, a la Facultad de Ciencias Marinas y al Instituto de Investigaciones Oceanológicas de la Universidad Autónoma de Baja California, por el apoyo financiero para la realización del presente trabajo. La tercera autora agradece a la Comisión de Operación y Fomento de Actividades Académicas (COFAA) del IPN, la beca otorgada. Carlos S. Mendoza preparó algunas ilustraciones.

\section{Literatura citada}

Aguilar-Rosas, M., L. E. Aguilar-Rosas y R. Aguilar-Rosas. 1998. Algas marinas de la región central de Quintana Roo, México. Polibotánica 7:15-32.

Aguilar-Rosas, L. E., R. Aguilar-Rosas, A. C. MendozaGonzález y L. E. Mateo-Cid. 2000. Marine algae from the Northeast coast of Baja California, México. Botánica Marina 43:127-140.

Aguilar-Rosas, R., L. E. Aguilar-Rosas, C. Mendoza-González y L. E. Mateo-Cid. 2007. Distribución latitudinal de las fases reproductivas de Lithothrix aspergillum (Corallinales, 


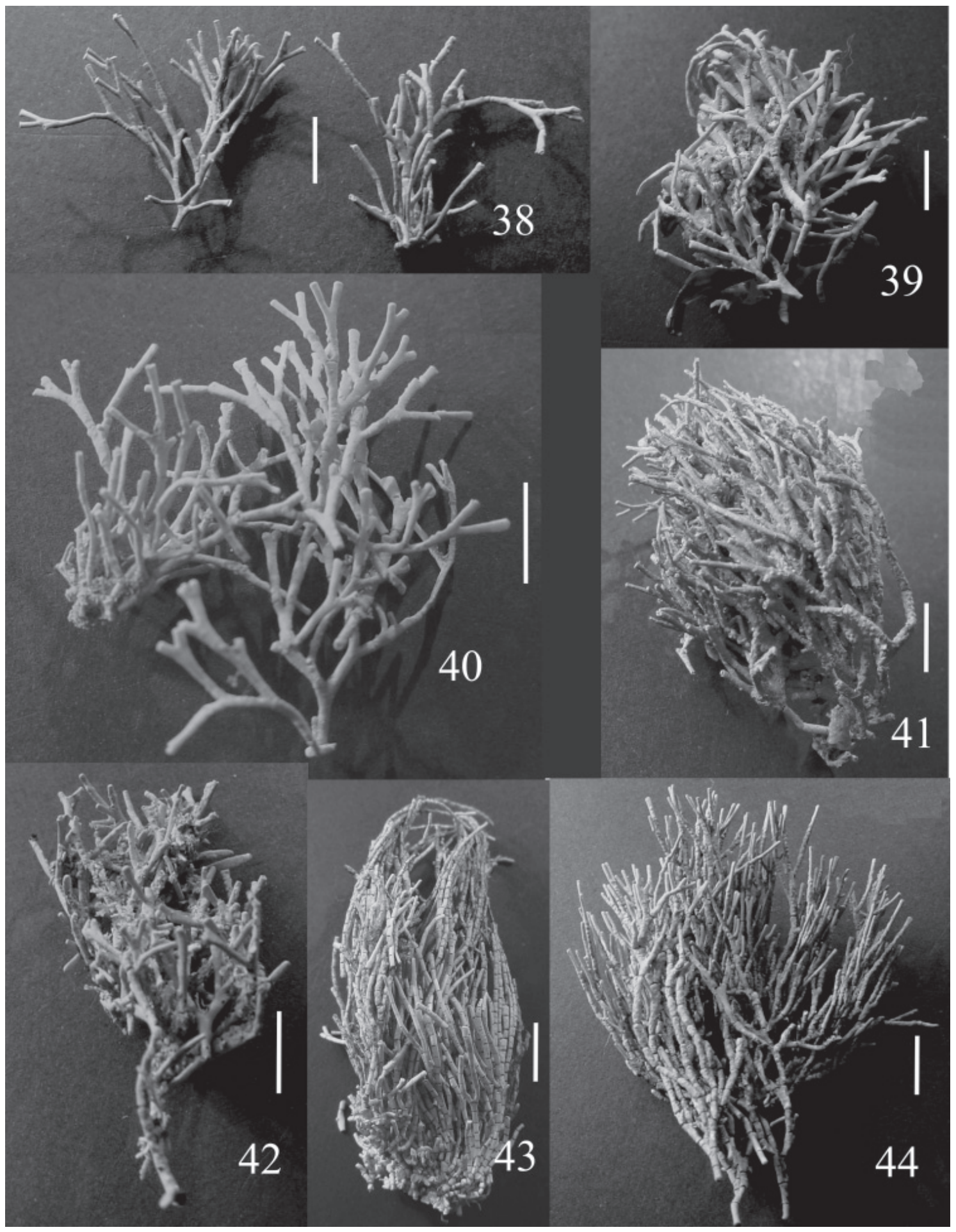

Figuras 38-44. Amphiroa beauvoisii. Variación morfológica de talos bisporangiales. 38. Ejemplar de Campo Turístico Vallarta, B.C. 31 de julio de 1996 (ENCB 12228). 39. Ejemplar de Puerto Peñasco, Sonora, 9 de marzo de 1997 (ENCB 15638). 40. Ejemplar de Las Palmas, Bahía Concepción, B.C.S. 23 de mayo de 1990 (ENCB 7996). 41. Ejemplar de Ensenada, B.C. 27 de julio de 1987 (ENCB 12211). 42. Ejemplar de Bahía Tortugas, B.C.S. 14 de junio de 1986 (ENCB 8913). 43. Ejemplar de La Manzanilla, Jalisco. 17 de noviembre de 1998 (ENCB 15641). 44. Ejemplar de Barra de Potosí, Guerrero. 25 de septiembre del 2007 (ENCB s/n). Escalas, 38-40 y 42-44: $1 \mathrm{~cm}, 41: 16 \mathrm{~cm})$. 
Rhodophyta) en la costa del Pacífico de México. Hidrobiológica 17: 7-74.

Anaya, R. G y R. Riosmena-Rodríguez. 1996. Macroalgas del arrecife coralino de Cabo Pulmo-Los Frailes, B.C.S., México. Revista Biología Tropical 44:903-906.

Dawson, E. Y. 1944. The marine algae of the Gulf of California. Allan Hancock Pacific Expeditions 3:1-450.

Dawson, E. Y. 1949. Resultados preliminares de un reconocimiento de las algas marinas de la costa Pacífica de México. Revista de la Sociedad Mexicana de Historia Natural 9:215-255.

Dawson, E. Y. 1951. A further study of upwelling and associated vegetation along Pacific Baja California, México. Journal of Marine Research 10:39-58.

Dawson, E. Y. 1953. Marine red algae of Pacific Mexico I. Bangiales to Corallinoideae. Allan Hancock Pacific Expeditions 17:1-239.

Dawson, E. Y. 1954. On correlation of marine vegetation with upwelling along the Pacific coast of Baja California, Mexico. In Proceeding of the Seventh International Botanical Congress, H. Osvald y E. Aberg (eds.). Almqvist and Wiksell. July 12-20, 1950. Stockholm. 827 p.

Dawson, E. 1959. Marine algae from the 1958 cruise of the Stella Polaris in the Gulf of California. Los Angeles County Museum Contributions in Science. Los Angeles, California 27:1-39.

Dawson, E. Y. 1961. A guide to the literature and distribution of Pacific benthic algae from Alaska to the Galapagos Islands. Pacific Science 15:370-461.

Dawson, E. 1966. Marine algae in the vicinity of Puerto Peñasco, Sonora, Mexico. Gulf of California Field Guide Series 1. University of Arizona, Tucson. 57 p.

Dawson, E. Y., M. Neushul y R. D. Wildman. 1960. Seaweeds associated with kelps beds along southern California and Northwestern Mexico. Pacific Naturalist 1:81 p.

Devinny, J. S. 1978. Ordination of seaweed communities: environment gradients in Punta Banda, Mexico. Botanica Marina 21:357-363.

Dolan, S. 2001. The use of medullary unit patterns of intergenícula and genícula in the taxonomy of Amphiroa (Corallinaceae, Rhodophyta). European Journal of Phycology 36:397-407.

Dreckmann, K. M., F. F. Pedroche y A. Sentíes-Granados. 1990. Lista florística de las algas marinas bentónicas de la costa norte de Michoacán, México. Boletín Sociedad Botánica de México 50:19-42.

Galindo-Villegas, I. J., J. A. Gamboa y K. M. Dreckmann. 1997. Estudio de las macroalgas marinas del Puerto de Salina Cruz en el Golfo de Tehuantepec. Pacífico tropical mexicano. Polibotánica 4:1-9.

Gómez-Gutiérrez, J., R. Palomares-García y S. HernándezTujillo. 2001. Community structure of zooplankton in the main entrance of Bahía Magdalena, México during 1996. Revista de Biología Tropical 49:454-558.

González-González, J., M. Gold-Morgan, H. León-Tejeda, C. Candelaria, D. León-Álvarez, E. S. Zaragoza y D. Fragoso. 1996. Catálogo onomástico (nomenclator) y bibliografía indexada de las algas bentónicas marinas de México, Cuadernos No. 34, Instituto de Biología, Universidad
Nacional Autónoma de México, México, D.F. 492 p.

Guiry, M. D. y G. M. Guiry. 2006. AlgaeBase version 4.2. Worldwide electronic publication, National University of Ireland, Galway. http://www.algaebase.org

Holmgren, P. K., N. H. - Holmgren. y L. C. Barnett. 1990. Index herbariorum. Part I. The herbaria of the world, octava edición. New York Botanical Garden, New York. 693 p.

Huerta-Múzquiz, L. y J. L. Tirado. 1970. Estudio florístico ecológico de las algas marinas de la costa del golfo de Tehuantepec. Boletín Sociedad Botánica de México 31:115-37.

Huerta-Múzquiz, L. y M. A. Garza-Barrientos. 1975. Contribución al conocimiento de la flora marina de las islas Socorro y San Benedicto del archipiélago Revillagigedo, Colima, México. Boletín Informativo Instituto de Botánica Escuela Superior de Agricultura, Universidad de Guadalajara 4:4-6.

Huerta-Múzquiz, L. y A. C. Mendoza-González. 1985. Algas marinas de la parte sur de la Bahía de la Paz, B. C. S. Phytologia 59:35-37.

Huerta-Múzquiz, L., A. C. Mendoza-González y L. E. MateoCid. 1987. Avances sobre un estudio de las algas marinas de la península de Yucatán. Phytologia 62:23-53.

Kützing, F. T. 1858. Tabulae phycologicae ...vol. 8. Nordhausen. II +48 p., 100 pls.

Lamouroux, J. V. F. 1812. Extrait d'un mémoire sur la classification des Polypiers coralligénes non entierement pierreux. Nouveau Bulletin des Sciences 3:181-188.

Lamouroux, J. V. F. 1816. Histoire des polypiers coralligénes flexibles, vulgairement nommés zoophytes. Caen. xxxiv + $559(560=$ errata $)$ p., xix pls.

Lemoine, M. P. 1929. Les corallinacées de l'Archipel des Galapagos et du Golfe de Panama. Archives du Museum di Histoire Naturelle Paris series G, 4:37-86 + (2).

León-Tejera, H. P. 1996. Caracterización florística del límite sur del Pacífico tropical mexicano: El litoral rocoso de Oaxaca. Tesis doctorado, Facultad de Ciencias, Universidad Nacional Autónoma de México, México, D. F. 146 p.

Littler, M. M. y D. S. Littler. 1981. Intertidal macrophyte communities from Pacific California and the upper Gulf of California: Relatively constant vs. environmentally fluctuating systems. Marine Ecology Progress Series 4:145-158.

Littler, M. M. y K. E. Arnold, 1982. Primary productivity of marine macroalgal functional-form groups from Southwestern North America. Journal of Phycology 18:307-311.

Martoja, R. y M. Martoja-Pierson. 1970. Técnicas de histología animal. Toray-Masson, Barcelona. $370 \mathrm{p}$.

Mateo-Cid, L. E. y A. C. Mendoza-González. 1991. Algas marinas bénticas de la costa del estado de Colima, México. Acta Botanica Mexicana 13:9-30.

Mateo-Cid, L. E., y A. C. Mendoza-González. 1992. Algas marinas bentónicas de la costa sur de Nayarit, México. Acta Botanica Mexicana 20:13-28.

Mateo-Cid, L. E. y A. C. Mendoza-González. 1994a. Estudio florístico de las algas bentónicas de Bahía Asunción, Baja California Sur, México. Ciencias Marinas 20:41-64.

Mateo-Cid, L. E. y A. C. Mendoza-González. 1994b. Algas marinas bentónicas de Todos Santos, Baja California Sur, México. Acta Botanica Mexicana 29:31-47. 
Mateo-Cid, L. E., I. Sánchez-Rodríguez, E. RodríguezMontesinos y Ma. M. Casas Valdés. 1993. Estudio florístico de las algas marinas bentónicas de Bahía Concepción, B. C. S., México. Ciencias Marinas 19:41-60.

Mateo-Cid, L. E. y A. C. Mendoza-González. 2001 (2003). Algas marinas bentónicas de la costa de Oaxaca, México. Anales de la Escuela Nacional de Ciencias Biológicas México 47:11-26.

Mateo-Cid, L. E., A. C. Mendoza-González, C. Galicia-García y L. Huerta-Múzquiz. 2000. Contribución al estudio de las algas marinas bentónicas de Punta Arena y Cabo Pulmo, Baja California Sur, México. Acta Botanica Mexicana 52:1-12.

Mendoza-González, A. C. y L. E. Mateo-Cid. 1985. Contribución al estudio florístico-ficológico de la costa Occidental de Baja California. Phytologia 59:17-33

Mendoza-González, A. C. y L. E. Mateo-Cid. 1986. Flora marina bentónica de la costa noreste del estado de Sonora, México. Phytología 60:414-427.

Mendoza-González, A. C. y L. E. Mateo-Cid. 1992. Estudio preliminar de las algas marinas bentónicas de la costa de Jalisco, México. Anales de la Escuela Nacional de Ciencias Biológicas México 37:9-25.

Mendoza-González, A. C. y L. E. Mateo-Cid. 1998. Avance de un estudio sobre las macroalgas marinas de Guerrero y Oaxaca, México. Ciencia y Mar 1:15-29.

Mendoza-González, A. C., L. E. Mateo-Cid y L. Huerta-Múzquiz. 1994. Algas marinas bentónicas de Mazatlán, Sinaloa, México. Acta Botanica Mexicana 27:99-115.

Moura, C. W. N. y S. M. P. Beauclair. 2005. O gênero Amphiroa (Lithophylloideae, Rhodophyta) no litoral do Brasil. In Monografías ficológicas, A. Senties G. y K. M. Drekmann (eds.). Universidad Autónoma Metropolitana- Iztapalapa, e Instituto de Botânica, Sâo Paulo 2:3-65.

Norris, J. N. y H. W. Johansen. 1981. Articulated coralline algae of the Gulf of California, México. I. Amphiroa Lamouroux. Smithsonian Contributions to the Marine Sciences 9:1-29.

Núñez-López, R., M. M. Casas-Valdés, A. C. Mendoza-González y L. E. Mateo-Cid. 1998. Flora ficológica de la laguna San
Ignacio, B.C.S., México. Hidrobiológica 8:33-42.

Ortega, M. M., J. L. Godínez y G. Garduño Solórzano. 2001. Catálogo de algas bénticas de las costas mexicanas del golfo de México y mar Caribe. Cuadernos 34. Instituto de Biología, Universidad Nacional Autónoma de México. 594 p.

Pedroche, F. F. y A. G. Sentíes. 2003. Ficología marina mexicana. Diversidad y problemática actual. Hidrobiológica 13: 23-32.

Riosmena-Rodríguez, R. y D. A. Siqueiros-Beltrones. 1996. Taxonomy of the genus Amphiroa (Corallinales, Rhodophyta) in the southern Baja California Peninsula, Mexico. Phycologia 32:135-147.

Sánchez-Rodríguez, I., M. C. Fajardo-León y C. OliveiroPantoja. 1989. Estudio Florístico estacional de las algas en Bahía Magdalena, B. C. S., México Investigaciones Marinas. CICIMAR 4:35-48.

Sánchez-Castrejón, E., R. Riosmena-Rodríguez y D. A. SiqueirosBeltrones. 1995. Nuevos registros de Berkeleya hialina (Naviculales: Berkeleyaceae) para el golfo de California, México. Revista de Biología Tropical 43:323-330.

Serviere-Zaragoza, E., J. González-González y D. RodríguezVargas. 1993. Ficoflora de la región de Bahía Banderas, Jalisco-Nayarit. p. 475-485. In Biodiversidad marina y costera de México, S. I. Salazar Vallejo y N. E. González (eds.). Comisión Nacional para el Conocimiento y Uso de la Biodiversidad y Centro de Investigaciones de Quintana Roo, México. 865 p.

Stout, I. y K. M. Dreckmann. 1993. Macroalgas bentónicas de Faro de Bucerías, Michoacán, México. Anales del Instituto de Biología, Universidad Nacional Autónoma de México, México, D. F. Serie Botánica 64:1-23.

Taylor, W. R. 1945. Pacific marine algae of the Allan Hancock Expeditions to the Galapagos Islands. Allan Hancock Pacific Expeditions 12:1-528.

Tyrell, B. y H. W. Johansen. 1995. Reproductive and regenerative strategies of Lithothrix aspergillum Corallinales, Rhodophyta) in southern California. Phycologia 34:39-44.

Yendo, K. 1902. Coralline Verae japonicae. Journal of College Science of the Imperial University of Tokyo 12:1-36. 